\title{
Efficient measurements of the diameter of the human artery using super-resolution imaging technique based on multi-scale wavelet analysis
}

\author{
S. Mekaoui ${ }^{1}$, S. Tchoketchkebir ${ }^{1}$ \& K. Ghoumid $^{2}$ \\ ${ }^{1}$ Département des télécommunications, LCPTS, USTHB, Algeria \\ ${ }^{2}$ ENSAO, Laboratoire d'electronique et des télécommunication, \\ Complexe Universitaire, Morocco
}

\begin{abstract}
The aim of the work presented in this paper is focused on the super-resolution technique for image processing in order to measure efficiently the diameter of the human artery. This work can find crucial applications in avoiding cerebral aneurisms if the physician has a good monitoring tool that can allow him to get this information early on the basis of the analysis of cerebral artery images. For this purpose, we have used a simulated artery home probe made of silicon and this model had been scanned by Phillips flat panel scanner (ALURA FD 20) with a resolution of $0.035 \mathrm{~mm}$. We have developed software based on the superresolution algorithm using the multi-scale wavelet analysis and able to reconstruct a high resolution image closest to the reality from a low resolution image. We have applied our image processing software to many images and have carried out a comparison with a super-resolution technique based on polynomial interpolation or B-splines interpolation and find out that our method yields better measurements of the artery diameter.

Keywords: super-resolution technique, image processing, discrete wavelet transform, multi-scale analysis, polynomial interpolation, B-splines interpolation.
\end{abstract}

\section{Introduction}

Super-resolution (SR) techniques consist of reconstructing a high resolution (HR) image from one or several low resolution (LR) images. The first objective of this technique is to correctly detect the contours of several objects in medical 
images. We here are proposing a modification of the SR technique based on the discrete wavelet transform and the well known multi-scale wavelet analysis to attain the same goal. Many problems and difficulties remained unsolved when good readability, high definition, good resolution of images specifically in medical imaging clinical interpretation for diagnosis and treatment are concerned. On the basis, of these image properties, it is crucial for the physician that the captured images reflect the real nature of the suspected disease. Usually, artifacts from unavoidable tremors and uncontrolled movements of the patient since the acquisition or problems generated by the equipment itself or the difficult conditions of acquisition like in the case of invasive based image analysis can partially or thoroughly blur the images making sometimes the diagnosis very difficult to the physician and sometimes impossible. Then the SR technique becomes a necessary tool not only for enhancing the visual quality of the images but also for extracting the hidden information that are contained in the real original images. So, we are rapidly limited by the image resolution. Indeed, if we increase the image resolution, the necessary interpolation will blur this image. In fact, it is impossible to generate the lacking information corresponding to a lack of details of the initial image. The SR methods are classified in three categories [1], such as: Interpolation methods; learning-based methods; methods based on reconstruction. The polynomial interpolation methods are based on the theory of up-sampling [2-4]. These methods are fast methods; nevertheless, they introduce many high frequency shifts on the details of the analyzed image and consequently blur it by fuzzy artifacts. The reconstruction methods [5-7], estimate a SR image by applying certain knowledge on the sampled image, for example; the edges smoothing. These approaches are generally designed to reduce the artifacts of high frequency range of high resolution (HR) images for contours detection. The performances of the HR images treated with the reconstruction methods depend on the nature and the geometric properties of LR images. In learning-based methods [8-10], detailed textures are highlighted by searching through a training of a set of LR/HR images. A drastic selection is made on the choice of the training image to avoid any blurring component $[11,12]$.

\section{Methods}

\subsection{General background}

Since a decade, the SR technique had launched in the research world a tremendous development of many algorithms for HR image reconstruction from one or a set of LR images. In the imagery domain, the word resolution is much concerned by the details fidelity and reliability. This resolution is also tied up to the image sampling rate that's to say to the number of pixels of the used sensor in the acquisition phase. In the algorithmic point of view, the single-image SR realizes the reconstruction of an $\mathrm{HR}$ image of size $(\mathrm{kH} \mathrm{x} \mathrm{kL)}$ from a single LR image. The application of the single-image SR technique is based on the theory of image oversampling (e.g. interpolation) 


\subsection{Polynomial interpolation}

In these approaches, we proceed to the convolution in time of a function $\mathrm{f}$ and a kernel function h:

$$
\mathrm{g}(\mathrm{x})=\mathrm{f}(\mathrm{x}) * \mathrm{~h}(\mathrm{x})=\int \mathrm{f}(\mathrm{y}) \mathrm{h}(\mathrm{x}-\mathrm{y}) \mathrm{dy},
$$

This corresponds in the frequency domain to the simple product of the respective spectrum of $f$ and $h$ :

$$
\mathrm{G}(\mathrm{u})=\mathrm{F}(\mathrm{u}) \mathrm{H}(\mathrm{u}),
$$

where: $\mathrm{F}$ and $\mathrm{H}$ are respectively the Fourier transform of $\mathrm{f}$ and $\mathrm{h}$.

Then, the ideal interpolation in this case is obtained with:

$$
h(x)=\operatorname{sinc}(x) ; \quad \text { and } H(u)=\operatorname{rect}(u) ; \quad \text { (In the frequency domain), }
$$

But, other rapid interpolation sub-methods are also used like:

\subsubsection{Interpolation of the closest neighbor}

In this approach the kernel function is:

$$
h(x)=\operatorname{rect}(x) ; \quad \text { and } H(u)=\operatorname{sinc}(u) ;
$$

\subsubsection{Quadratic interpolation}

In this approach, among many kernel function formulas that have been proposed is this reported in reference [13] which is particularly used today in this context and whose expression is given below:

$$
h(x)=\left\{\begin{array}{lr}
-2 x^{2}+1 ; & \text { if }|x| \leq \frac{1}{2} \\
x^{2}-\frac{5}{2|x|}+\frac{3}{2} ; & \text { if } \quad \frac{1}{2} \leq|x| \leq \frac{3}{2} \\
0 &
\end{array}\right.
$$

Then the interpolation function is obtained with the following kernel function expressed in the frequency domain:

$$
H(u)=\frac{6 \sin (\pi u)-6 \sin (3 \pi u)-\cos (\pi u)+\cos 9}{(2 \pi u)^{2}} ;
$$

\subsubsection{Cubic interpolation}

In this category of interpolations, there exist numerous techniques yielding numerous kernel functions. We will retain the following family:

$$
\begin{cases}h(x)=(a+2)|x|^{3}-(a+3) x^{2}+1 & \text { if } \quad|x| \leq 1 \\ h(x)=a\left(|x|^{3}-5 x^{2}+8|x|-4\right. & \text { if } 1 \leq|x| \leq 2 \\ h(x)=0 & \text { otherwise }\end{cases}
$$

where: a: is a parameter. The value $\mathrm{a}=-1 / 2$ is always retained. Then, the interpolation function is obtained with the following transfer function expressed in the frequency domain:

$$
H(u)=\frac{18-24 \cos 2 \pi u+6 \cos 4 \pi u-\pi u(2 \sin 2 \pi u-\sin 4 \pi u)}{(2 \pi u)^{4}} ;
$$




\subsection{Wavelet background}

\subsubsection{Continuous wavelets transform (CWT)}

The continuous wavelets transform of a finite energy function $\mathrm{f}$ is given by:

$$
C_{f}(a, b)=\int_{-\infty}^{+\infty} f(t) \cdot \psi_{a, b}^{*}(t) d t=<f, \psi_{a, b}>;
$$

where: $\mathrm{a} \in R^{+*} e t \quad b \in R$, and $\quad \psi_{a, b}(t)=\frac{1}{\sqrt{a}} \psi\left(\frac{t-b}{a}\right)$, denote the wavelet family.

\subsubsection{Discrete wavelets transform (DWT)}

The discrete wavelet transform is simply a discrete adapted form of the CWT which encounters now the discrete dyadic scale of the variable a. Thus, the wavelet function becomes:

$$
\psi_{n, m}^{*}(t)=a_{0}^{\frac{-m}{2}} \psi\left(a_{0}^{-m} t-n b_{0}\right) \text {; Where: } a_{0} \text { and } b_{0} \text { are constants. }
$$

Then, the discrete wavelets transform is then defined by:

$$
g(t)=\sum_{n \in Z, m \in Z}<f, \psi_{n, m}^{*}>\psi_{n, m}^{*}(t) ;
$$

where the symbol: $<>$; denotes the inner product.

\subsubsection{D-wavelet multi resolution analysis}

The principle of the 2D-wavelet multi resolution analysis is to represent the analyzed image at several levels of resolution. The image of interest (image of an artery) is supposed to be sampled prior to the analysis. Then, this image is projected in the space vector $\mathrm{V}_{0}$. This space is then decomposed in two series of subspaces $\left(\mathrm{V}_{1}, \mathrm{~V}_{2}, \mathrm{~V}_{3} \ldots \mathrm{V}_{\mathrm{j}} \ldots\right)$ and $\left(\mathrm{W}_{1}, \mathrm{~W}_{2}, \mathrm{~W}_{3} \ldots \mathrm{W}_{\mathrm{j}-1} \ldots\right)$. The first series of subspaces contains all of the approximations of our image and is characterized by the existence of a series of approximations coefficients, whereas, the second series contains all of the details of our image and is characterized by the existence of a series of details coefficients. The approximations of our original image also allow the extraction of low frequency components while the details permit the extraction of high frequency components of our original image. The approximations spaces as well as the details subspaces are included in one another. Moreover, the operation of extracting both frequency components is done recursively under the following assertion:

$$
V_{j-1}=V_{j} \oplus W_{j} ;
$$

\section{Algorithm descriptions}

\subsection{Image acquisition}

As cited above and for testing the robustness of our super resolution algorithm, we have used a homemade probe of a Silicon based artery. This model had been scanned using a Philips flat panel (alura FD20) with a resolution of $0.035 \mathrm{~mm}$.

We have taken many images and have constituted a data base of medical images named DICOM and stored it for future use. 


\subsection{Images treatment}

In this section we present the method of the image treatment which consists in using the wavelets transform to solve the problem of image super resolution from LR (Low Resolution) images. This process contains four essential steps, namely: image oversampling; smoothing of oversampled images; internal artery diameter calculus.

\subsubsection{Super resolution process (SR)}

The single image SR process principle is based on the images oversampling techniques (interpolation). The latter is based on introducing intermediary samples between two or several current and known samples. This operation is realized whenever we have to convert an image in a pre-defined geometry.

\subsubsection{Algorithm steps}

\subsection{2a) First step}

In this step, we consider that the original image is sampled in respect to Shannon's condition so that we will be able to reconstruct any part of it from a discrete series of samples representing a fraction or the whole original image. Then this discrete image is filtered so that it yields an image whose spectrum is compatible with the new desired sampling. After what, we sample again the same image at the desired points (points of interests). Nevertheless, this principle faces hard and difficult hardware constraints to implement it, so one has to find other substituting solutions. This is done by combining the reconstruction with filtering to build a new single filtering. This can also be achieved by replacing the construction functions by more compact functions like polynomial functions allowing finite and infinite summations.

\subsection{2b) Second step}

Once we get the HR image with the different polynomial filters, the image details follow the characteristics of the interpolation function. Then, the idea consists in using the multi resolution analysis based on stationary wavelets in order to process to a smoothing of this HR image and collect all of the details that follow the characteristics of the original image. At the first scale, the wavelet transform will simply extract the most refined and very sharp details. At the second scale, the details extracted will be less refined and less sharp and hence, the process is stopped when we get an image that is completely smoothed.

\subsection{2c) Third step}

Once we obtain the smoothed image after a certain number of iterations, we calculate both diameters (internal and external) to build up a final image that has the desired geometric characteristics. Then, for the need of comparison, we use the original image as a reference. We deliberately have chosen to weaken the resolution of these images and then have applied our algorithm in order to carry out a comparison. 


\section{Results}

Figure 1 given hereafter, illustrates the original scanned image of the section of the artery with the Phillips scanner. The size of the image is 556x556 pixels with an internal diameter $\mathrm{d}$ of about $4.315 \mathrm{~mm}$.

Figure 2 gives a series of three low resolution images that have been deliberately weakened with a different reduction factor $\mathrm{f}$. Then, we apply to the same images our implemented algorithm super resolution wavelet based process and we get the results illustrated in Figure 3, given below. We can see clearly the enhancements and the calculated values of the internal diameters of the artery.

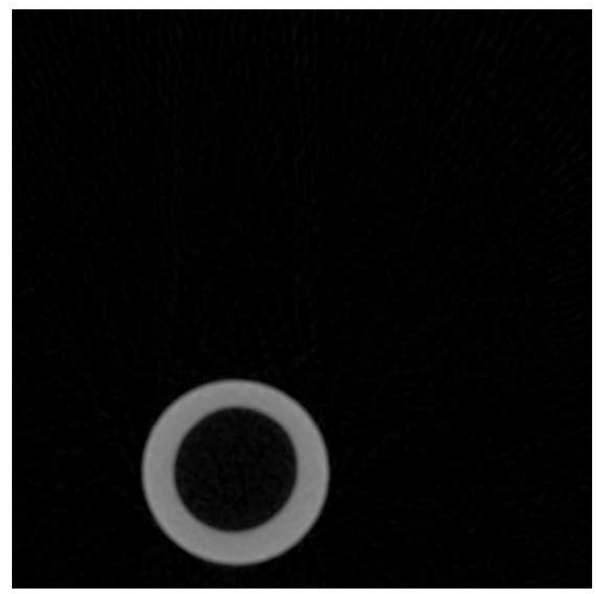

Figure 1: Original image of size 556x556 with an internal diameter $\mathrm{d}=4.315 \mathrm{~mm}$.

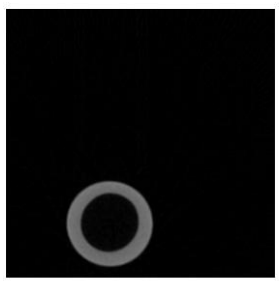

(a)

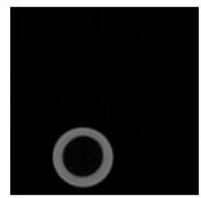

(b)

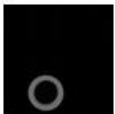

(c)

Figure 2: (a) LR image of size $278 \times 278$ pixel ( $f=2$ ), (b) LR image of size $139 x 139$ pixels $(f=4)$, (c) Same image of size $70 x 70$ pixels $(f=8)$. 


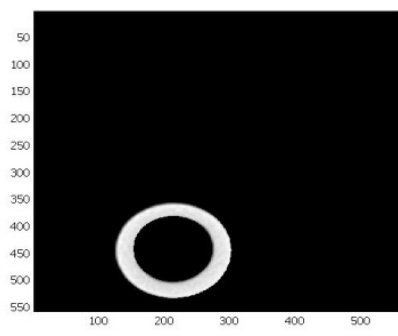

(a)

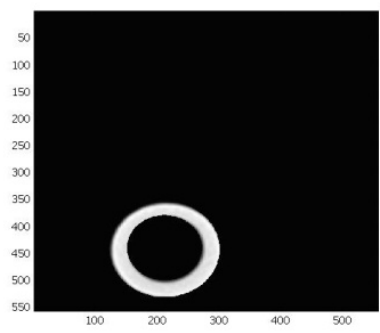

(b)

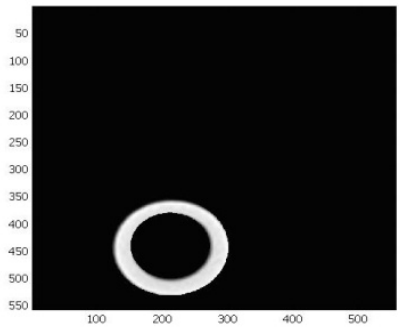

(c)

Figure 3: (a) HR reconstructed image of size 556x556 ( $\mathrm{f}=2, \mathrm{~d}_{\text {int }}=4.312 \mathrm{~mm}$ ), (b) HR reconstructed image of size 556x556 ( $\left.\mathrm{f}=4, \mathrm{~d}_{\text {int }}=4.307 \mathrm{~mm}\right)$, (c) HR reconstructed image of size $556 \times 556\left(\mathrm{f}=8, \mathrm{~d}_{\mathrm{int}}=4.312 \mathrm{~mm}\right)$.

\section{Discussion and validation}

To test the efficiency of our algorithm, we have considered the original images as referring images and we applied our super resolution algorithm based on the wavelet multi resolution analysis on 40 low resolution (LR) images with different resolution factors (after the down sampling). We them, compared the performances of our algorithm on 20 LR images having with different factors of super resolution to two others such as; Bilinear function SR with circular smoothing and Bi-cubic function SR with circular smoothing. The results are compared in Table 1, given hereafter.

Table 1: $\quad$ Results of SR-wavelets, SR bilinear and SR bi-cubic with a circular smoothing and a comparison of four parameters $\left(D_{\text {mean }}, \sigma^{2}\right.$, e and Acc.) versus Super Resolution factor f (SR factor).

\begin{tabular}{|c|c|c|c|c|c|c|c|c|c|c|c|c|}
\hline \multirow{2}{*}{$\begin{array}{c}\text { SR fact. } \\
\mathrm{f}\end{array}$} & \multicolumn{4}{|c|}{ Wavelet based SR } & \multicolumn{3}{c|}{ Bilinear SR with circular smoothing } & \multicolumn{3}{c|}{ Bi-cubic SR with circular smoothing } \\
\cline { 2 - 14 } & $\begin{array}{c}\text { Dmean } \\
\text { in (mm) }\end{array}$ & $\sigma^{2}$ & e & Acc & $\begin{array}{c}\text { Dmean } \\
\text { in (mm) }\end{array}$ & $\sigma^{2}$ & e & Acc & $\begin{array}{c}\text { Dmean } \\
\text { in (mm) }\end{array}$ & $\sigma^{2}$ & e & Acc \\
\hline 2 & 4.3121 & 0.0026 & 0.0061 & 0.069 & 4.313 & 0.0046 & 0.0101 & 0.045 & 4.3136 & 0.0039 & 0.0092 & 0.034 \\
\hline 4 & 4.3070 & 0.0024 & 0.0056 & 0.187 & 4.308 & 0.0039 & 0.0093 & 0.150 & 4.3092 & 0.0042 & 0.0098 & 0.139 \\
\hline 8 & 4.3220 & 0.0029 & 0.0068 & 0.159 & 4.325 & 0.0042 & $\mathbf{0 . 0 0 9 9}$ & $\mathbf{0 . 2 4 3}$ & 4.3264 & $\mathbf{0 . 0 0 1 8}$ & 0.0044 & 0.261 \\
\hline
\end{tabular}


In table 1, the parameters that had been compared are simultaneously, the mean value of the internal diameter, $\sigma^{2}$ the variance of the same value, the error ' $e$ ' and Acc the ratio of accuracy. These parameters had been calculated for the three kinds of SR algorithms on the basis of 20 LR images and it is worth noting that the error had been taken as $\mathrm{e}=2.35^{*} \sigma^{2}$, the nominal diameter $\mathrm{D}_{\mathrm{n}}=4.3151 \mathrm{~mm}$ and the accuracy ratio had been calculated with the following formula:

$$
\text { Accuracy }=\left[\frac{D_{n}-D_{\text {mean }}}{D n}\right] * 100 \text {, with } D n=4.3151 \mathrm{~mm} ;
$$

In order to validate the results carried by our algorithm (based wavelets SR algorithm), we have compared the error parameter for the three techniques and have drawn the curves of error versus the SR factor $f$ chosen and have done the same for the accuracy ratio. The curves are shown in Figures 4(a) and 4(b).

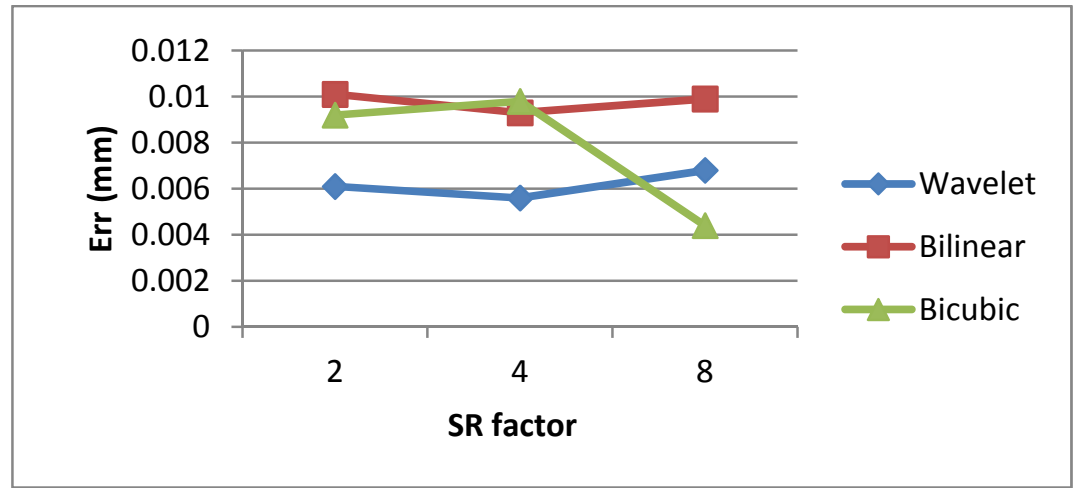

(a)

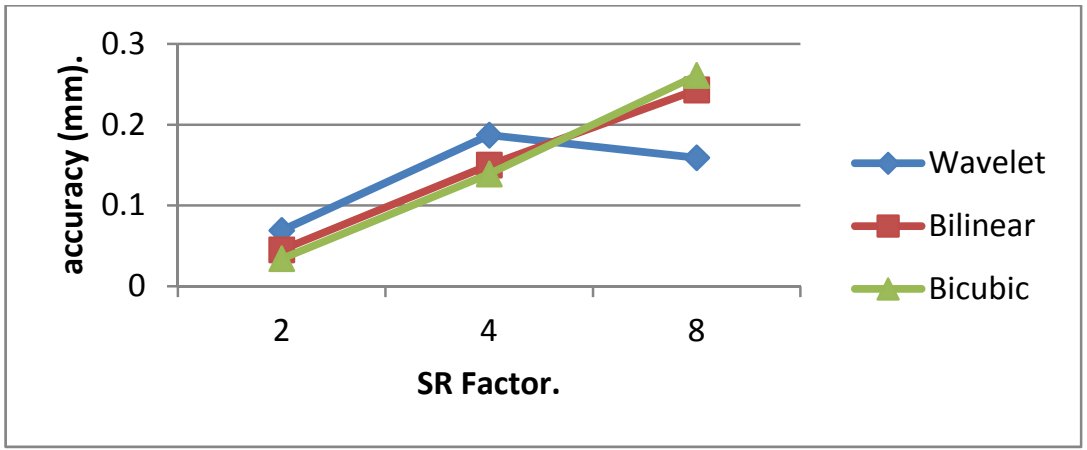

(b)

Figure 4: (a) Compared error versus SR factor for the three SR techniques. (b) Compared accuracy versus SR factor for the three SR techniques. 
From Figures 4(a) and 4(b) we can observe that the wavelet transform yields results with an error inferior to $0.007 \mathrm{~mm}$ for the three values of the SR factor whereas both other techniques (bilinear and bi-cubic)yields results with errors that are greater than $0.095 \mathrm{~mm}$. However, the bi-cubic at the level 8 yields results with an error that is less than $0.005 \mathrm{~mm}$, but, on the other hand its accuracy ratio is the biggest compared to the other super resolution algorithms (twice the wavelets-based SR algorithm). The results of the super resolution algorithms that are based on polynomial functions and smoothing filters vary when we change the interpolation function and the smoothing filters.

\section{Conclusions}

The main aim of our work was concerned by solving a medical imaging problem which addresses the precise detection of the contours of the specific objects in a medical image. Our specific interest in this paper is the detection of the exact internal diameter of an artery. This is particularly important to the clinician when this study targets the cerebral arteries to prevent aneurysms. This software can serve as a very useful tool to the physician if this detection on the real time image can be integrated in his monitoring system. The super resolution is a delicate problem in medical image processing. This technique allows image corrections and good quality image reconstruction even from poor quality original images. In this paper, this technique had been combined to the multiscale wavelet analysis in order to improve it and it has been shown that compared to other SR algorithms using polynomial function, our SR-algorithm based on multi-resolution wavelet had earned the challenge. Also, as it has been shown that in this technique, the characteristics of an HR image were following those of the LR image. Whereas, in the case of the SR techniques based on polynomial function, the HR image follows the characteristics of the interpolation function and the smoothing filter.

\section{References}

[1] J. Sun et al. "Image super-resolution using gradient profile prior". In CVPR, pp. 449-455, 2008.

[2] H. S. Hou and H. C. Andrews "Cubic splines for image interpolation and digital filtering”, IEEE Trans. Acoustics, Speech and Signal Proc., ASSP26:508-517, 449.

[3] X. Li and M. T. Orchard, "New edge-directed interpolation” IEEE Trans. on Image Processing, 10:1521-1527, 2001. 449, 450, 455, 456.

[4] W.S. Tam, C.W. Kok and W. C. Siu. A modified edge directed interpolation for images. Journal of Electronic Imaging, 19(1), 013011: 1-20, 2010.

[5] Q. Shan et al. "Fast image/video up-sampling". ACM Transactions on Graphics (SIGGRAPHASIA), 2008. 449, 451.

[6] K.W. Hung and W. C. Siu "New motion compensation model via frequency classification for fast video super-resolution” In ICIP, 2009, 449. 
[7] C. Liu et al. "Face hallucination: Theory and practice" 75(1):115-134, 2007.

[8] W. T. Freeman et al. "Example-based super-resolution" IEEE Comput. Graph. Appl., 22(2):56-65, March 2002.

[9] H. Chang et al. "Super-resolution through neighbor embedding” In CVPR, vol. 1, pp. 275-282, 2004.

[10] R. Fattal "Up sampling via imposed edges statistics" ACM Transactions on Graphics (Proceedings of SIGGRAPH 2007), 26(3):95:1-95:8, 2007.

[11] K. I. Kim and Y. Kwon "Example-based learning for single image superresolution and jpeg artifact removal” Technical Report 173, Max Planck Institute, August 2008.

[12] N. Dodgson "Quadratic interpolation for image re sampling”, IEEE Trans. on Image Processing. Vol. 6, n9, p. 1322-1326, septembre 1997.

[13] B. Chalmond "Eléments de modélisation pour l'Analyse d'Images" vol. 33, Springer Verlag, Mathématiques et Applications. 2000. 